\title{
La educación como sistema
}

por ANTONIO J. COLONH

A Alejandro Sanvisens con profundo agradecimiento

\section{(1. INTRODUCCION}

lil termino "Sistema" aplicado a la liduc acion supone plantear una perspectiva de estudio del fenomeno educasional de acuerdo con los dictados metodológicos patrocinatos por la 'Teoria ciemeral de sistemas.'

1 la leoría General de Sistemas desanoblada de manos de l. V. Mertalanffy como metodologia de ha ciencia se hallit formuladia de forma conerefa por su autor en los siguientes

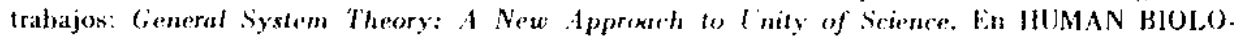
(jY. V. York 1951. Diciembre, Pge 303 a 361.

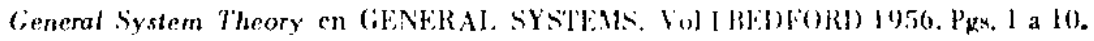

Theorie Generale des Sivstemes. Paris 1973. Dunod.

P'uede romsultarse además:

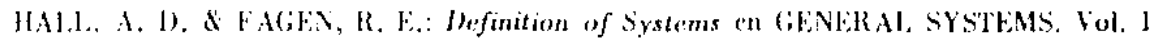
lied ford 1950. Pgs. 18 a 29.

BELR, S: Cibernética y Administracion. Hexieo 1972. C. li. C... A.

BOLILINA, K.: Cencral Systems Theory: The Sketeton of Sience en MANAGEMENT SCIF N(L. N. York, Abril 1956. Pgs 197 a 20108.

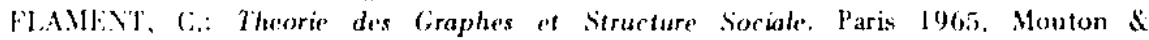
Gautbiers Villars.

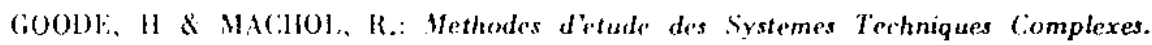
Paris 1963. Dunod.

HARl, l. F.: L analyse des Systemes Paris $19 \% 2$. Iunod.

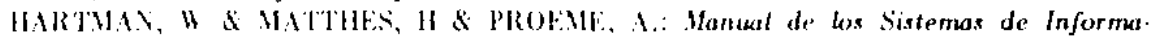
cion (2 T.). Matrid 1973. Vidic. Paraninfo. lica Téanica Philips.

HII.L. J. R.: Toward a Scionce of Oranization. Oregon 19613. Oregon Intiversity Press. li. l. (i. ti. H, E.

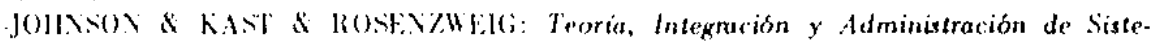
mós. Néxior 1970. limusa Hiley.

1.AYKARO. V.: Sistemas y Procedimientos. Hexieo, 2." Vidic. 1972. Fdit. Diana.

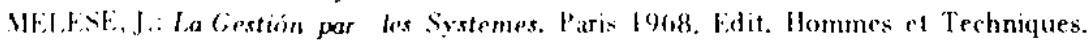

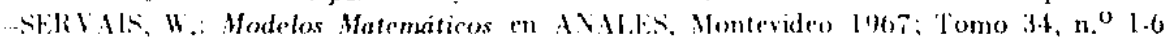
l'g. 61 a 688.

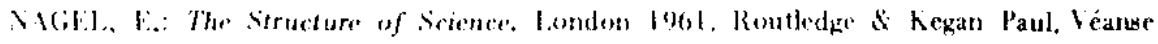

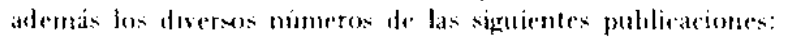

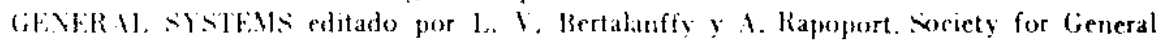

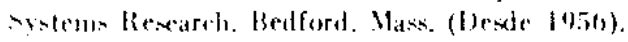

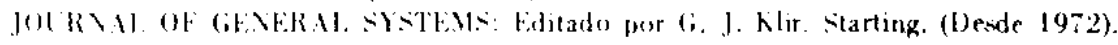


De hecho la tradicion sistemisa en liducacion es mínima y realemente actual. Esto hase que el cuerpo de dertrina sistimica que sobre la biducación se posece no sea de las mismas proporciontes que cl recopilado en otras disciplinas humano-sociales tales como la propia sociologia, la liconomía, la Organización. Ein este sentido debo afirmar las dificultades halladas en fundamentar bibliográlicamente el trabajo que presento, maxime cuando he pretendido no alejarme de las fuentes pedagógicas. Para ello he utilizado una bibliogratia interdisciplinar dentro de los límites de las Ciencias de la liducacion que contempla obras de Sociologia de la liducacion, Pedagogía (abemética, Organizatión liseolar, Pedagogria Comparada... ete. ${ }^{2}$ lodo ello con ánimo de presentar lo más ampliamente posible: un esquema que, al menos con valide\% metodologica, asiente los puntos esenciales sobre el que deberá basarse algún día un estudio completo de la liducacion desde una perspectiva sistémica.

fin Fispaña el [0r. Mlejandro Sanvisens, caledrático de la llniversidad de Barcelona publicó en 1972 y an 1973 dos trabajos que de alguna forma inaguraban ésta

2 Los Trabajos que patrocinan ma metodologia sistemica a la hora de estudiar la Educación no se distinguen por su abundancia. El propio Bertalanffy en Theorie conerale des Systemes. (Paris 1973) de: un repertorio bibhogrático de más de $\$ 20$ títulos solo cila los siguientes estudios de carácter pedagogico:

MACCIA, E \& STEINJK, li \& MACCLA, G. S.: Development of Educational Theory Deririent from Threo Educational Theory Modols. P'roject 5 -0638. Columbus Ohio. The Ohio State Resarch Foundation 1966.

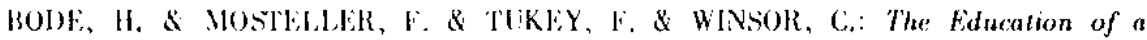

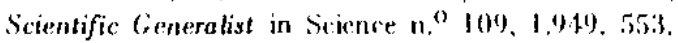

MATHER, K. F.: Objetives and Tature of Integrative Studies. in MAN CDiRRENTS IN

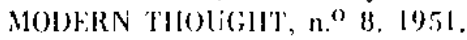

BERTALANFY, 1. V.: Philesophy of Science in Scientific Education. Sc:lF.VT. MONIIIL. n, n."77, 1953.

A ellos pueden sumarse algunos olros trabajos tales como:

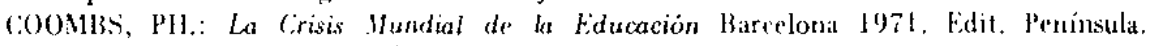
Colec. Historia, Ciencia, sociedad, n, 32.

FRANK, H.: 1spectos Cientifices Téorices y Organizatorio Ciberneticos de la Pedagogia Cibernética. Folia Humanistica, T. VH, X. 8. Diciembre 1969.

HOPPEK, E. (Edit. by): Reading in the Theory of Edutational System. London 1971. Hutehing Sion \& Co. Publ.

-..KAUFAAN, R. A.: Phnificación de Sistemas Fducativos. Ideas Básicas Concretas México 1973. Fdit. Trillas. Se refiere esentialmente a cut'stiones l)idácticas.

PARSONS, T: The Sithool Cikss as a Social System some of its Function in America

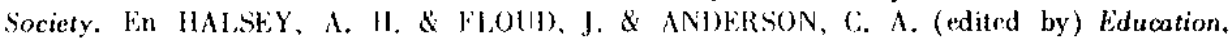
Fconomy and Society. S. York 1961. Free Press.

RICF, A. k.: Iniversidad Moderna. Madrid 1972. Serv. Public. del Ministerio de lidueacion y ciencia.

Rollsyoski. 1. J.: The systems dproach to fiducution and Training. London 1970) Kogan Piste.

Como se comprenderí sólo me reficro a las obras que patrocinan un enfoutue sistemico de la Fducacion basamentado en la Teoria General de Sistemas. Vo se toman et consideración un sinnumero de trabajos que dexte perspectivas diversas y con significados muy personales se refieren a la bduacacion como sistema. 


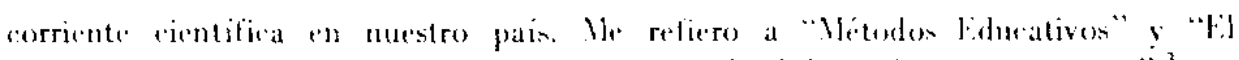

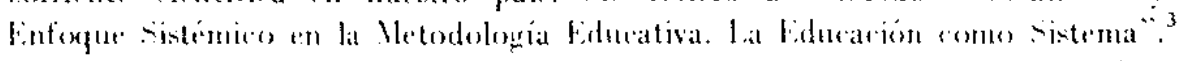

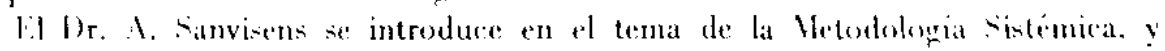
tal eomo hace constar en la bibliografia que cita, a partir, nutre ofros de Bertalanfly y Mesarovir. Su roncepto de sintema es realmente amplio sifendo considerado fomo una realidad compleja intercomexionada. Sin embargo al matro de la leoria Cientral de Sistemian no és para el Br. Nejandro Sanvisens punto de partida sino verdadera meta intuda a lo largo de mis de veinte años. Fen electo es a partir de 1956 chando inicia una serie de publicaciones que supomen mola singhadura inedita en el pithorama intelectuat-humanistion espanol. Su primitiva vocación filoséfica le impele at busear un esquema válido, explicativo de la realidiad, al mismo tiempo que rechatza los contextos al uso que la l lniversidad espanola le ofrecia an esta epora. Nejándose de sistemats filosolicos recién innortardos (existemetalismo), logra conectar eon una corriente de penamiente realmente incdita en mestro pais. la Cibernétiea lo lleva a integrarse cerea de coneepeiones estructuralistats que aplieará preferentemente al campo de la socelologia y de la Pedagengia sin olvidarse de otras

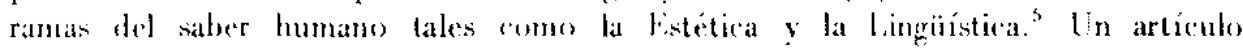

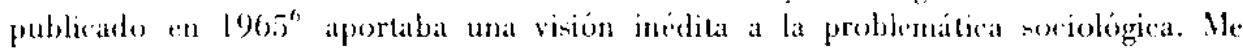
refiero al astudios de la kelación enmo paso previo para formular mal teoría de la Integration. A. Sanvisens llamalsa Teoria de la lntegration a le que Bertalanfy

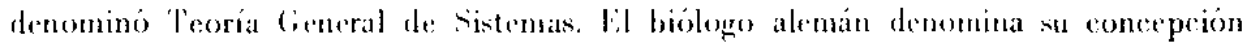
hajo premikas deseriptivas y 1. Sanvisens lo hacia ron la consernentia a con el objetivo que de herhoconcurre en la leoria de sistemas. Si Bertalantfy busea con ella la unidad de la Cieneia, hanvisens se refería a la "Integracion". Necesariamente entonces, y como) un feliz determinismo, su lator debia ineidir en la lemática sistemiéa a la que ha aportado aspectos realmente interesantes. Coneretame dire que ha ofrecido una de las

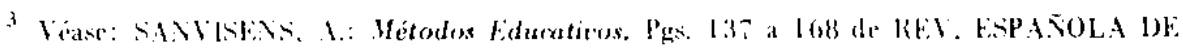
JeEngogita, n. 118 . Abril-junio 1972.

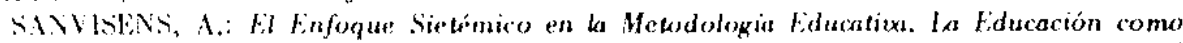

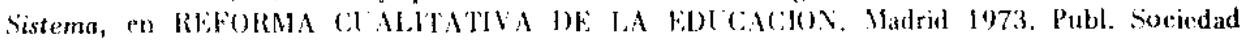

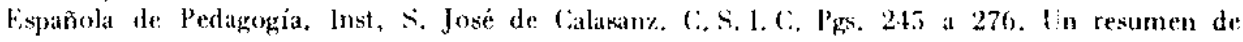
este trabijo pucde consultarse en Resumenes de Ponencias y Comunixariones del $l$ Congreso Nacional de Pedagogia. Burgos 1972. Hijos de si. Rodriguez.

Impirandose en la ponencia del Dr. Sanvisens, presentada en el ritado Congreso de Pedagegia, F. Hascort realizó su Pesis Doctoral auncque apliranto su estudio al campo de la

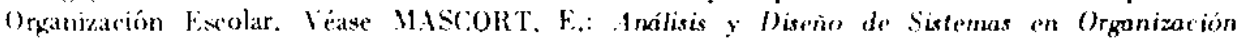
Liscolar. Barcelona 1974. Iniversidad de Barcelona. lacultad de Filosofia y letrax. Dírigida por -l Dr. Fernándè Huerta. (luedita).

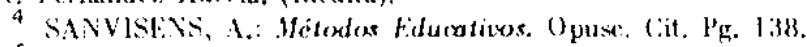

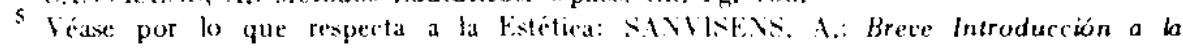

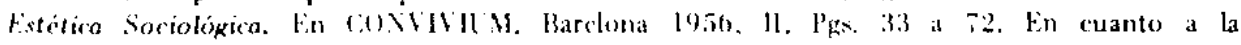

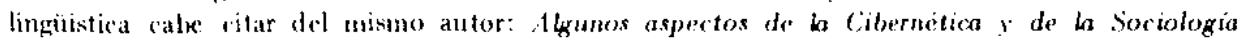
lingüsstien. Barcelona 1971. Asociación de Ingenieros Industriales.

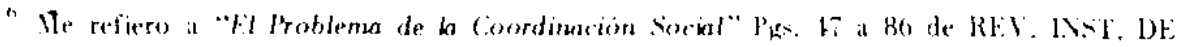

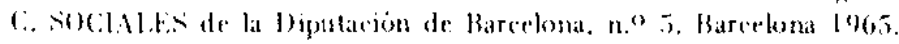


taxonomials más perferetas referida a los sistemas. Por otra parte ha aplicado la metodología sistemica a un campo tal como of de la liducacion carente de una tradición en este sentido: además de ésta aplicación ha entresacado un método parit estudiar los sistemas educativos (Analisis y l)iseño dis Sistemas) concretade en las rapas siguientes: Planteamiento, Sclectividad, funcionalidad, Representacion, Votacion. Operatividad (desarrollo algorítmico). Búspe la de relaciones. Contrastacióno aplicación Analógica, Aplicabilidad y Litilizatción.

La caracteristica delinitoria de lo que A. Sanvisens llama "realidad compleja interomexionada" (Sistema) es la posesion de dos aspertos inter-independientes que el denomina Realidad Operativa y Realidad Ontica. I a Realidad Operativa en cualquier sistema vieme dada por su funcionalidad y la categoría esencial de su realidad por su estructura. lite este sentido lodo Sistema sera una listruetura Funcional que vendrí implícilamente definida por la Coordinación o adecuación y ordenation relacionante de codos los elementos que lo forman.

Pista coordinación o arglutinación que posibilita la fenomenotogia de un Sistemat he especifica en tres planos:

fil plano Interno del sistema en el que se realiza la integratión básica y funciomal.

H.l plano Internotixterno que solicita del medio la información y sustentacion al mismo tiempo que expresil su reaccion frente al mismo.

lif plano fixterno que proporciona estimulación, compensación y posibilitat (ion de equilibrio. ${ }^{7}$

A continuarión pasaré a exponer las bases en las que se atsentarí la concepción sistémica de la fiducación que patrocinamos. De hecho partimos - y nos referimenos mas explicitamente a través de nuestro trabajo de las aportaciones realizadati al tema por el Dr. A. Sanvisens.

\section{CONSIDIRACIONES PRIVIAS AI. HEFOQLI: SISTIEMICO DI: LA FUUCACIÓN}

Antes de estudiar la liducación desde una perspectiva sistemica es necesario dislucidar unos aspectos previos e introducir unos puntos de partida si es que no se: quiera restionar ya de principio el enfoque que patrocinamos.

Consideramos a la bducación como subsistema focial por lo que se ha de demostrar que realmente la fiducación en un elemento -complejo- de indole social. lista ha de ser nuestra primera tarea si queremos que el posterior desarrollo toruga validez.

l.a Fducacion ha side fruto del crecimiento en complejidad que ha sufrido la Soriedad. Reruérdese que una de las leges confignadoras de la sociedad se formula dievende que lodo crecinsiente en complejidal supone una mayor diferenciacion tanto a nivel estructural come a nivel funcional. Exactamente es lo que ocurrió

7 liumdamentado en lbjobm. 


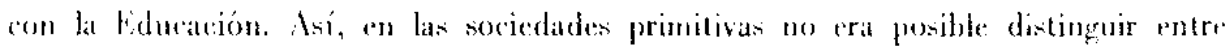

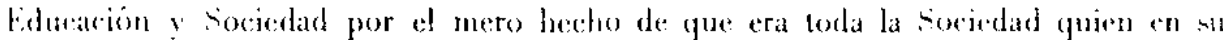
dinámica procesual natural educaha a sus miembros. fil motivo de esta situarion

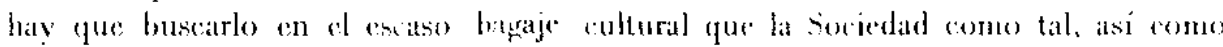
sus micubros individualnante considerados, poselan. De esta sitiacion se desprende que cualquier miembro de la colectivitad temia rapacidad de realizar la mision

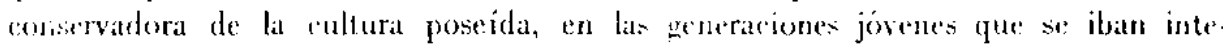

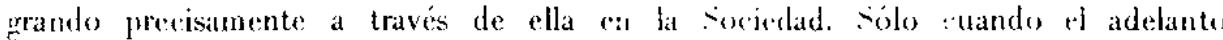
derneo de la humandad se amplia. y con ello las necesidades sapienciales bánica para la propia supervivencia de los miembros de la colectividad, del núcleo de

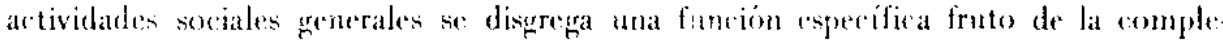
jidad alcanzada por la cociedad. Lista fimcion $n 0$ es otra que la transmisora de conocimintos e integration de las generaciones jóvenes en los valores y norman que rigen para sus mayores. Desde este momente lat propia sociedad crea sil ristema liducativo que será entonese el encargato y el responsable de cumplimentar los objelivos gae le encomienden.

En un principio a Sistema fiducativo fue +minententente familiar-tribal y en este sentido, ligado plenamente a la organization fermeral social. Fl momento que marca la separación y la distinción de lo educativo con la estructura general social se halla en la aparioion de los primeros centros especificos de educacion " de formación de lán nuevas gemeraciones, o sea en la aparicion de la liscuela. Puede entonces decirse que "las escuelats no aclían como algo aparte... no son entidades "xtrasocietariats; se encuentran insertas en el sistemal socjal y no por encima o sobre [.]" *

Sin embargo parece que nos hallamos ante una contradiceion andque no sea así. Ll fenómeno ducalivo, a pesar de dame en la soociedad y gracias a la Sociedad, es un fenomeno individual, de base psiquica, astentado en las relacioness docente-disente o colucador-educando. Lat sociedad posibilita la situacion educativa ereando el clina, proporeionando los medios y estalileciendo los objetives y la orientación que debe poseer. ${ }^{9}$ La edueacion fue en este momento de disgregatción funcional, un proceso social especilico que inicio sin desartolo con la Escuela.

Con el tiempo se vio que la lisenela no podia rumplimentar de forma plena la misión que tenia encomendada. Fsta mision conocida bajo la denominación de "liducación" consistia y consiste aun igualmente hoy en dial desde una perspectiva sociológica, en los siguientes objetivos:

- Asimilación de chltura, paulas y normas sociales.

Transmisión de cultura, pautas y normats sociales de una generación a otra.

l'reparación de las nuevas gencraciones para lat vida (en su más amplio sentilo) en la Sociedad.

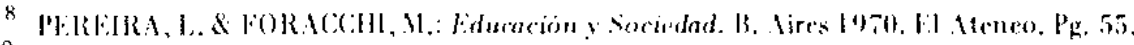

9 Ibidim. 
() como dice J. Bouspuets "I al educación es el proceso por el ctal la sociedad se transmite de generacion en generarion "so

Esta insuficiencia funcional educativa de la fiscuela fue debicta a las mismas causas que: posibilitaron su aparicion o seat la complejidad social. Téngase en cuenta -y es el propio Bousuet quien lo dice ${ }^{1}$. que la Fiscuela como fruto de la ampliación de la fenomemologria seseial, surge con la ciudad. lat laudad como elemento representativo de la complejidad sorial necesitit más de la éscuela que las comunidades maless. Asi, al arrecentarse las necesidades de la vida urbana, la eseucla como sistema educador no podial cumplimentar los objetivos que se le: habian ecomendado. Ial listuela pronto se vio rodeada de instituciones paralelas cuya misión era y sigue siendo la de cumplimentar los objetivos que ella misma, de una forma plena y eficaz dejaba de cumplir. l)esde las instituciones gremiales a los liducational Shoping Centers toda una gama de elementos socictales complimentan la misión educativa que la complejidad social diferenció de sin más íntima estructurit. Hoy en diat, y visto el avance y desarrollo de la sociedad, toda ella pretende ser educiativa y educadora; tanto ss asi que hace años ya surgió un cuerpo de opinión que pretendia la supresión de la escutla por su inclicatia cara al mondo de mestros días: "I a Socieckad tiene yee ser una sociedad educada para progrewar y erecer y aun sobrevivir". 2

Con ello llegamos a una de lats premisas que consideramos básica atarar para continuar con el tema. Me setiere a que la tiscuela con el tiemper ha llegado a ser sólo una parte del Sistema bilueativo. la escuela no es más que un subconjunto de una entidal educativa total mutho más amplia que comprende la familia, los Mass Media, el medio de: vida, de trabajo, lats relaciones de conviveneia... ete. ". Ouiero expresar ron ello que tel Sistenta liducativo no sólo abarca la Institución liscolar sino que de él participa también la familia, la iglesia, los medios de comunicación de masas, la pablicidad, al medio de trabajo, los partidos políticos, la pertenemeia at una dase social, las propias relaciones humantas... etc. Todo ello forma y conforma el macro conjunto social que poses ma misma finalidad tal como es a biducacion. Por otra parte, y porque la funeion educadora se asienta en anos elementos en interacción dehidamente oryanizados respecto de un fin. "proceso evolutivo determinado, al conjunto de Instituciones o aspectos sociales que exclusiva, o conjunta-

io BOLSOLET. J.: Fionomía Politica de ta Fiducación. Madrid 1960 last. de Estudios Políticos. Pg. 21 .

11 Ibidem. Pgs. 13 y sigs.

12 DRUCKKR, P.: Ia Rewhatión Lducxatim. Pg. 210 a 221 de ETZION, A. \& ETZIOVE, E. Los Cambios Sociales, Méxiro 1968, F. C. ., l'p. 216.

Véase además con lo que se relitre a las ciudad biducativa:

FAlRE, E. \& COLABOR IDORES: 1prender a Ser. Madrid 1973. Alianza l'NLSCO, así como las obras de P. Cieodman. I. Iltich. li. Remer por lo gue respecta al movimiento de la descolarización.

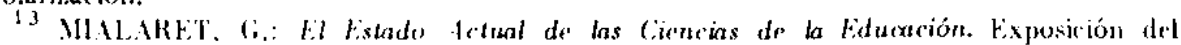

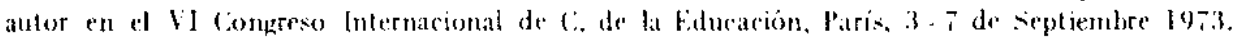


mente con otros objetivos, poseen la misión educadora, se le drinomina sistema Edurativo.

lintrexaco una series de conclusiones que considero fundamentales para la clarificacion del concepto de Sistema fiducativo. De: una forma ronereta dire que fumos testablecido los siguientes e importantes puntos:

1. La fiducación es un fenomeno social en cuanto se da en toda Sociedad y is un dato inferente y ligado al hecho mismo de su existencial conservación $y$ desenvolvimiento. ${ }^{14}$

2. la fiducation no solo se da en la Fescela sino en una serie de institucio. nes, circunstancias $y$ aspectos también de indole social que poseren al igual que: aquellas una misión educadora.' ${ }^{5}$

De lit primera premisa podemos comeluir afimando:

-()ue la liducación está integrada en el Sistema tocial.

Por parte de la segunda:

Quir lo "educativo" abarca un amplio campo de lo Sorial.

A partir de aqui ya se vislumbra la posibilidad de definir a la liducación (omo Sistema.

[besde el momente en que la fiducación esta integrata en al sistema Social, participa de su dinamicidad $y$ de su capacidad procesual y evolutiva. la liducación se halla desde ef nomento que es un fenomeno que se da con la Sociedad inmersa en una realidad cambiante con capacidad de influir $y$ ser influida. sin permanencia dentro de esta realidad cambiante salla en la rapacidad adaptativa y en la ultratstabilidad que forzosamente ha de posecer.

Por otra parte, la liducación forma un eomplejo o lotadidad conslituido por edementos con misión educadora y que por lanto, por participar del mismo objetive y referirse al mismo fin, han de hallarse en interrolación. Puede decirse monomes que la tiducación forma un Sistema (en cuanto subsistema de la sociedad) por ster una totalidad compleja y dinamica formada por la interredación de elemenlos con capacidad processual respecto de un fin.

Fif Sistemal lidurativo es un subsistema def ristema Social formado por la interretación dinamica con capacidad procesuat de la totalidad de instituciones, clementos, unidades complejos y aspertos sociales o de índole social cuya misión o función social so total o parcialmente educativa, considerando aquí el concepto "liducacion" en su mas amplia expresión como conjunto de pautas a transmitir a las nuevas generationes para que se de el mantenimiento y el progreso de la vida humana y social.

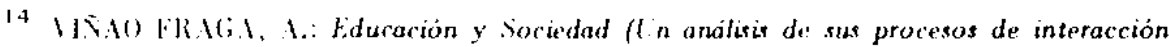

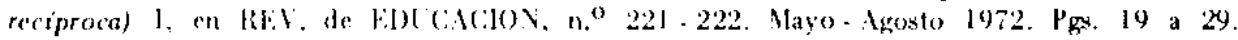
Veas Py. Pth.

Is lin el sominario Internacional de Cirncias de la fiduciacion celebrado en Barcelona del

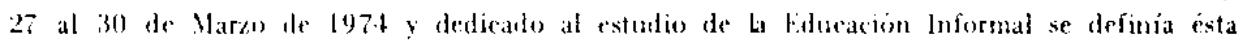
como "Fducacion renthida a trates del ambiente". 
A pesar de haher detimitado el concepto de Sistema liducativo quitero dejar constancia tal como hace el 1)r. A. Sanvisens de la limitacion que aún hoy por hoy posec el enfoque sistémico aplicado a la fiduación:

"linfocar la biducán como sistema no implica de buenas a primeras, que "todo" en la liducasión sea perfectamente sistemático, ni metnos aún, que" esté sistematizado con el rigor fuc la l'eoría de Sistemas - que trata precisumente de desarrollarse en mestra época -.. exige en el plano cientifico", 16

\section{EL SISTEMA IDDUCATIVO: APORTACIONISS A SU LSTUDIO}

\section{1. Una Definición}

Cada Sociedad, tal como vimos, posec un Sistema liducativo que es impuesto a sus miembros de un modo consciente, determinante y rígido; tanto es así que se ha llegado a decir que puede conocerse toda una estruetura social conociendo su Sistema Educativo. ${ }^{17}$

la liducación como Sistema forma parte ded Sistema Social a nivel de sulsistema. Pertenece al tipo de Sistemas Socio-Culturales en el sentido que l'. Sorokin da al témino, en los que inciden una estruclura y dinámica personal, social, y cultural basada en los procesos de interaccion compuestos por tres lactores tales como:

\section{Seres Humanos.}

Significaciones, Valores y Normas.

- Acciones extertits y fenómenos materiales.

A. Sanvisens ${ }^{*}$ ve también el Sistema liducativo como Sistema Socio-Cultural en inter-relación con todos los procesos sociales y culturales (linguísticos, arlísticos, científicos, técnicos...) y en otro sentido religioso, económico, político... ete.

El estudio del Sistema liduativo, según el mismo autor puede realizarse sincrónicat y diacrónicamente o sea, en su aspecto situacional, en un estado dado, $y$ en su sentido procesual o de secuemia temporal histórica.

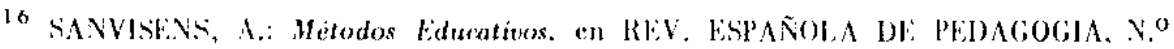
118. Abril-Junio 1972. P'gs. 137 a $168 . \mathrm{P}^{2} \mathrm{~g} .137$.

17 MERNANBliz, E.: Funcionalidad y Folución de los Sistemas Pedagógicos. P'gs. 5 a 58

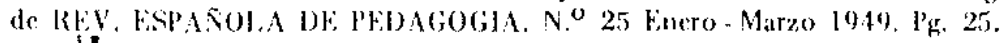

is SANVISENS. A. El enfogue Sistémico an la Metodologúa Educatiox. Ia Fiducación

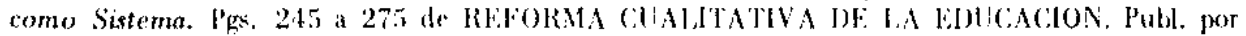

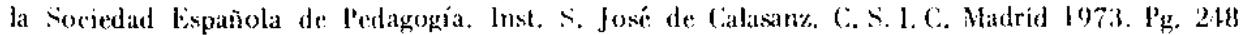
y sig. Ver también en "Rosumenes de Ponencias y comunicaciones del $V$ congreso $N$. de Pedag. Sies. If (Ponencia del mismo título) Burgos 1972. Hijos de Santiago Rodrigue.. Pgas. Ifol a 163. 


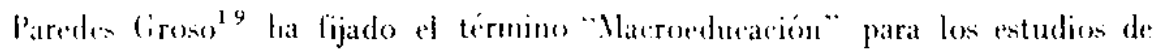
la "estruetura y funcionamiento del sistema educacional "sea de la liducacion en el contexte social en el que se mueve". Grizás tengia que hater constar que la Natrociacacion no ha de lomarse como otra perspertiva de estudio dentro de los gue se ratalogan en las (aencias de la liducacions sera en todo caso una pestura o un punte de vista de estudio de la Sociología de la liducacion, o una parte de tla: la que estudiatse a la fiducacion como sistema social. fin este sentide nuestro estudio del sistema liducation se integra ya dentero de la Sociologria de la lideracion en edanto he considerado al sistema fidurative romo sub-sistema del sistema serial.

lit Sistema lidurativo está formade por efementos formales (la encolarización en todos sas grados, gue da lagar tal como vimos a la que podria llamarse Instilueión liseolar a Subsistema liseolar en cuanto dependiente del sistema tiducalivo) y por lo que podriamos llamar etementos informales o posililidades educativas que las diversas instituciones, o el Sistemtit social an grenteral, ofrece como una democratización de lats formas educativas, o comos un intento de extender más amplianente las nomati, valoress significareiones preeninemestes.

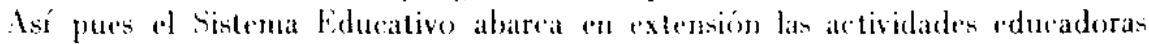
yere se desarrollan en la Familia, (irupo Parentat, agrupación de Maestros y l)iscipuJos (c)emento formal del Sistema liducativo: Subsintema liscolar), grupos locales de antgos. grupres de edad, grupos sexuales, grupos escolites, grupos estatales, grupos de lenguaje, grupos religiosos, grupos politicos y grupos laborales asi como los

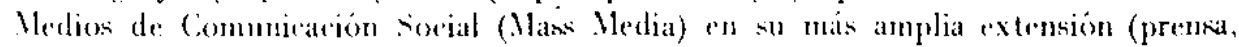
libros, cine, radio, encerertos, teatro... etc.). I esta descripeión del sistema liducativo original de P. Sorokin ${ }^{20}$ quiero añadir addemats los siguientes elementos: l'ertenencia a una rase social determinada, las redaciones humanas en su mas amplio sentido: el turismo y los medios of formats de vida actual no mencionadas (otros tipes de ocios, la publicidad, el ambiente arbano... etc.). Con ello conereta-

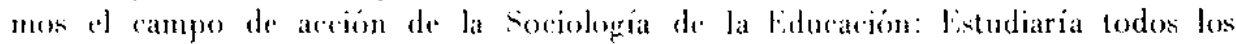
chementos anteriores as comos los procesos que ton ellos romeurren y que hacen posible desempenten una fumeión edurativa ronsiderando dicha función en su más

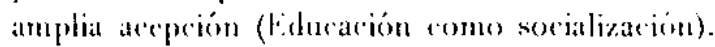

la bidacacion desde el momento en que se considera enmosistema se ha de ver como lotalidad de elementos en interaceion. Hice al respecte Ph. Coombs, ${ }^{21}$ uno de los piomerese en el estudio sistemice de la liducación, que ésta forma un sistema en el que cada parte ha de emitir sus propias señales para advertir ni la aleción conjunta se realiza de forma sitifactoria " no. lin resumen y de aewerdo

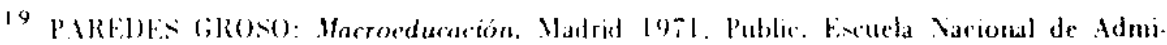

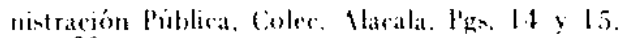

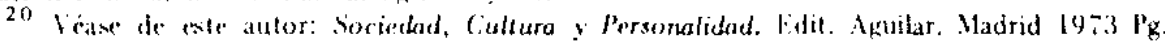
(16).

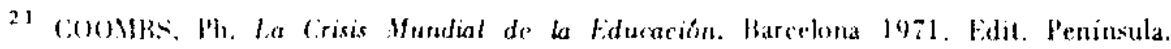

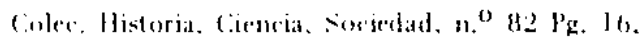


con lo dicho concretaré el concepto de Sistema tiducativo en la siguiente definición:

Fil sistema fiducativo es un subsistema del Sistema Social formado por la interacción dinámica con capacidiad procesual respecto de unos objetivos, de la totalidad de instituciones, elementos, unidades, grupos $y$ aspectos sociales o de índole social que posean total o parcialmente una lunción educadora, o sea que permitan a una población deterninada formarse (socializarse) mediante la internalización de un eleneo cultural y de pautas y normas de acción asi como de significaciones de acorde con los valores sociales establecidos.

\section{2. Tipo de Sistema}

lil Sistema fiducativo puede concebirse como un Sistema que posea las caracretísticas de los Sistemas $\Lambda$ biertos, Cerados y de Comunicación.

\section{2. 1. Sistema Abierto}

Ha sido A. Sanvisens ${ }^{22}$ yeien a puesto las bases definitorias del Sistema liducativo cono Sistema thierto al afirmar que se "inserta en un medio humano y socio-cultural complejo y multiforme en chya funcionalidad queda integrada la liducación como "determinante-determinado" influyendo decisivamente en dicho medio y siendo a su ve\% influida decisivanuente por el".

Por ser un Sistema Abierto poseerá unas entradas (inputs) y unas salidas (autputs) hacia la Sociedad.

las entradas que el Sistema Fiducativo posee de la Sociedad han sido determinadas a nivel general por 'T' Parions al decir:

"Imput por parte de la comunidad consiste en al apoyo de: la comunidad a la bducación como lunción en la decisión de mantener o mejorar el nivel de capacidad de la población".23

A su vez estas entradas son concretadas por Ph. Coombs ${ }^{24}$ según el esquema siguiente:

$\begin{array}{ll}\text { Conocimientos ya existentes } & \text { FINIS DE LA HDUCACION } \\ \text { Valores, Metas } & \text { CONTENIDOS } \\ \text { Población y Mano de obra } & \text { FSTUDIANTES } \\ \text { cualificada } & \begin{array}{l}\text { PROFESORES } \\ \text { RELACIONES HUMANAS IORMADORAS }\end{array} \\ \text { Producto economico e } & \text { IINANZAS } \\ \text { ingresos } & \text { ECONOMIA }\end{array}$

22 SANVISENS, A.: El Enfoque Sistémico en la Metodología Educativa. La Educación como Sistema. Opuse. Cit. Pg. 249. Ver además del mismo autor: Ponencia del mismo tílulo en Resumen de ponencias y comunicaciones... Opusc. cit. Pg. 161 y 162.

Ver también: Hétodos Fducativos Opuse. (it. Pg. 139.

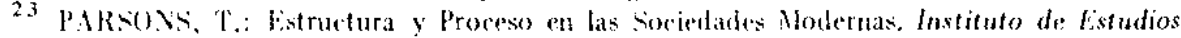
Polticos. Madrid 1900. Pg. 76 .

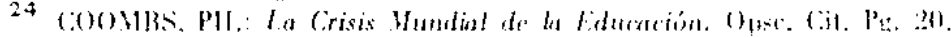


Las salidas del Sistema fiducativo hat ja la Sociedad vendiám dadas por les individuos educados con lodas las secuelits que este nuevo aporte de información neguentropía - posee para la Sociedad.

()ue un Sistema biducativo es un sistema abierto se demuestra además por la fenomenologia que puede observarse en la realidad. Por una parte la "conformación" del Sistema liducativo nunca es perfecta estando siempre en constante devenir, intentando ajuslarse a las exigencias del medio (social) que le rodera. Por otra parte las fronteras del conocimiento gracias a los avances de la fducación están cambiando continuamente influyendo en la sociedad, y esta en su transformación, incide de forma renovadora en el Sistema fiducativo.

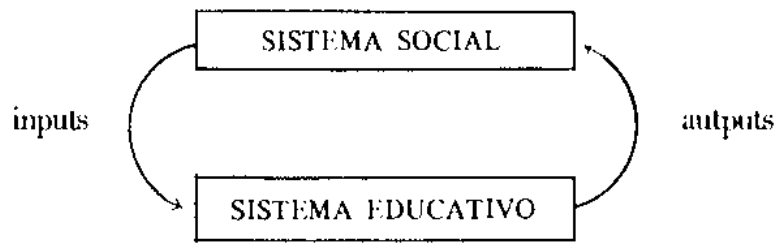

\section{2. 2. Sistema Cerrado}

Al mismo tiempo el Sistema fiducativo, y siguiendo al Dr. A. Sanvisens, es lambién un sistema cerrado:

"Mirado en sí mismo bajo el aspecto de: la retroacción positiva o" negativa (aumentativa o correctiva) que la educación ofrece, en relación con el medio y com su propia efectividad, el sistema educativo se ofrece como cerrado en cuanto a la dotorminación de su estruetural".

bisto quiere decir que el Sistema liducativo tiene capacidad reguladora propia correctora la optimizante - o sea que es capaz de administrar lo más eficazmente posible los contenidos de sus entradas, de acuerdo con los objetivos que tiene propuestos.

bil Sistema liducativo es pues abierto en ruanto a sus conexiones con el exterior y cerrado en cuanto a sus posibilidades retraactivas o de regulación.

A. Sanvisens sintetiza los dos aspectos antes mencionados el sistema educativo como sistema abierto y cerrado a partir del gráfico que inchuimos en la página sigruiente. 27

25 Anadido personalmente puesto que considero que en ta formulación primitiva de Ph. Coombs se hace exchusivamente referencia al sistema fiscolar.

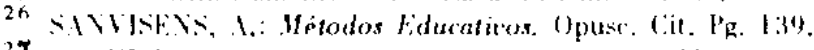

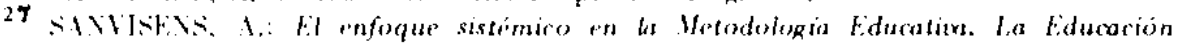
como sistema. Opuse. Cit, j'z. 2il. 


\section{2. 3. Sistema de Comunicación}

Además el Sistema liducativo es un Sistema de Comunicacion. A. Sanvisens ${ }^{28}$ y J. C. Agulla ${ }^{29}$ están de acterdo en ello puesto que la romunicación es ef mecanismo mediante el cuat se cumple: la función social del proceso educativo.

\section{JI, SISTHMA FDUCATIVO (OOMO SISTEMA ABIFRTO - CERRADO SEGIT EL DR. A. SAVISEAS}

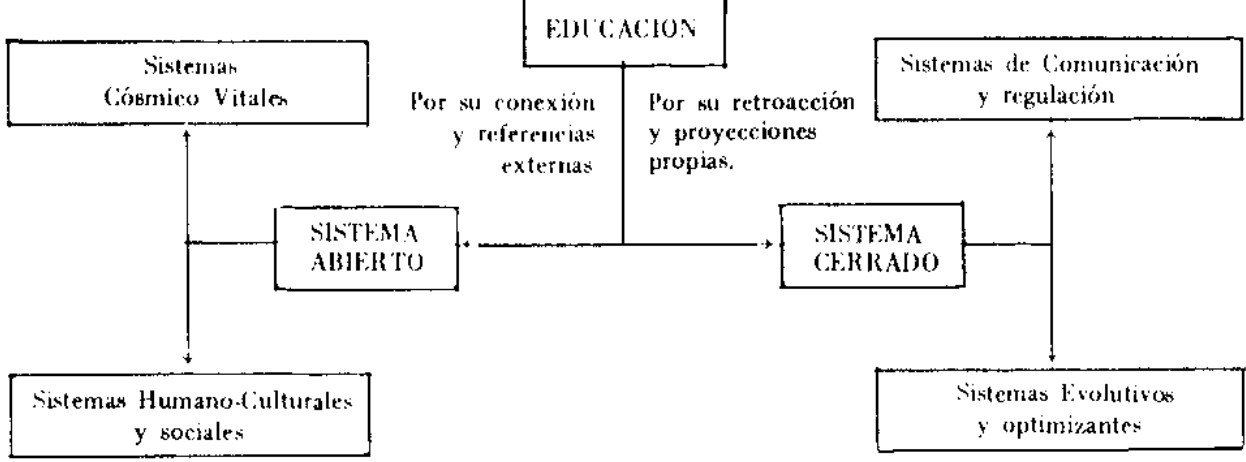

Puede decirse que el Sistema liducalivo:

-rin cuanto a sus relaciones en el Sistema Social es

- SISTIMMA NBIERTO)

lin cuanto a sus relaciones consigo mismo es

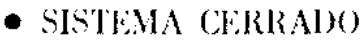

-...in cuanto a sus relaciones con los sujetos objetos de su función

- sistema dofi cominic:acion

Como sistema de Comunicación y considerado a modo de cjemplo la misión educadora de la lglesia tendriamos:

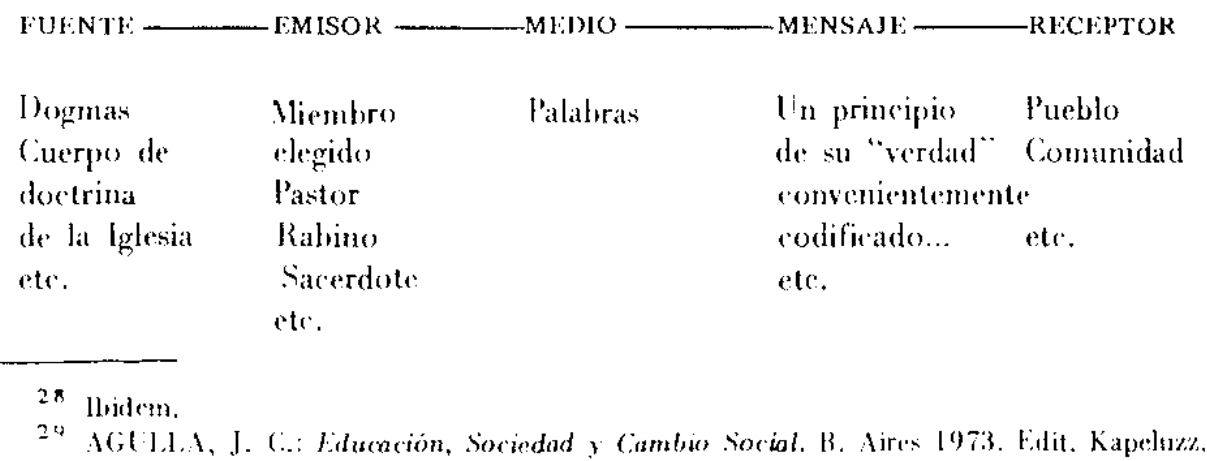
Pa. 95. 
En ell caso de la Institución liseolar neria:

I. UF NOH: HMSOS MII)IC MFNSAJI Kl:C:PJOR

\begin{tabular}{|c|c|c|c|c|}
\hline l'rogrraunas & I) exente: & Palalıra & Contenidos & Mlusneses \\
\hline Riagruaje & & lexnolagrias & $d e$ & \\
\hline cultural & & & aprendizaje & \\
\hline
\end{tabular}
de: la sion.

E) Sistema fidueativo cumplimenta su nision funcionatodo como si feese un Sistema de Comunicación. Ahora bien. todo sistema de Comunicalión no es tal en tanto ne tenga el recepler capacidad de emitir : de tomar af primitivo emisor como ronseruente receptor. lillo supone que un Sistema de Comunicación para serlo, ha de posear aceion de ida y vuelta. o seit capacidad de retroaceion. lil

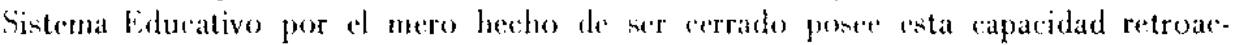
liva que tambien la apliea resando, de aruerdo eon su fun ion, se determina como Sistemat de Comumicación.

Ani, atl los ejemplos propuestos, la cafjacidad retroactiva se hallará en el Sistemat rompanador gue establecerá comexiones antre las respuestas que dan los sujetos en sa práctica religiosi o en en aprendizaje de: un tema, y el paradigna entablerido romo el deseado por la fuente: (la leglesia en el primer caso o el nivel exigrido culturalmente en el segundo), posibilitindese monten la capacidad correclora del ristema en su posterior acción y de aruerdo con los resultados anteriormente obtenidos.

\section{2. 4. Sintesis: Concepto de Sistema del Sistema Fiduratio}

De forma gritica y de acuerdes con lo dicho hasta ahora y por supuesto adecuado al enfoque tripartito, diseñanes il sistema biducativo tal como aparece en el esquema de la página sigutente.

lin dicho grálico, la luente tal como se ve en comsecuencia de la cultura y de los valores sociales que romo "entratas" darins pie al sistema para formar su demento "comparador de objetivos". lil limisor es una parte del propio sistema liducativo; el mensije es fruto de la elahoracion de contenidos que el sistema lidurative realiza de aluerdo eon sus entradas: el medio puede estar integrado en el

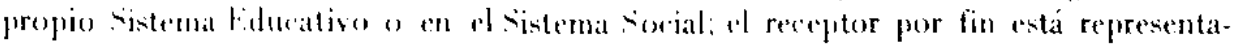
do por la loblación.

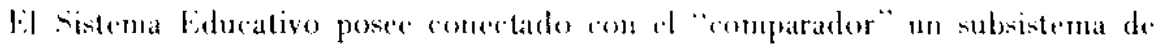

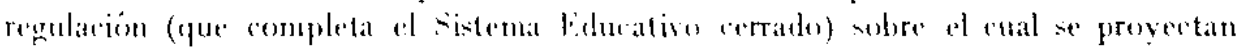

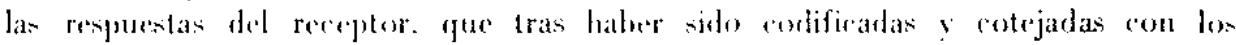

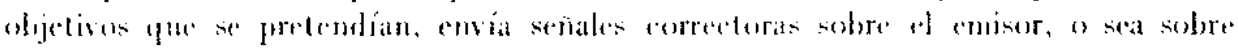
a propios sintemat Fibuation. 
$\ddot{\approx}$

MODELO TRISISTEMICO DEL SISTEMA EDUCATIVO.

SMA. SOCIAL

SMA. SOCIAL.

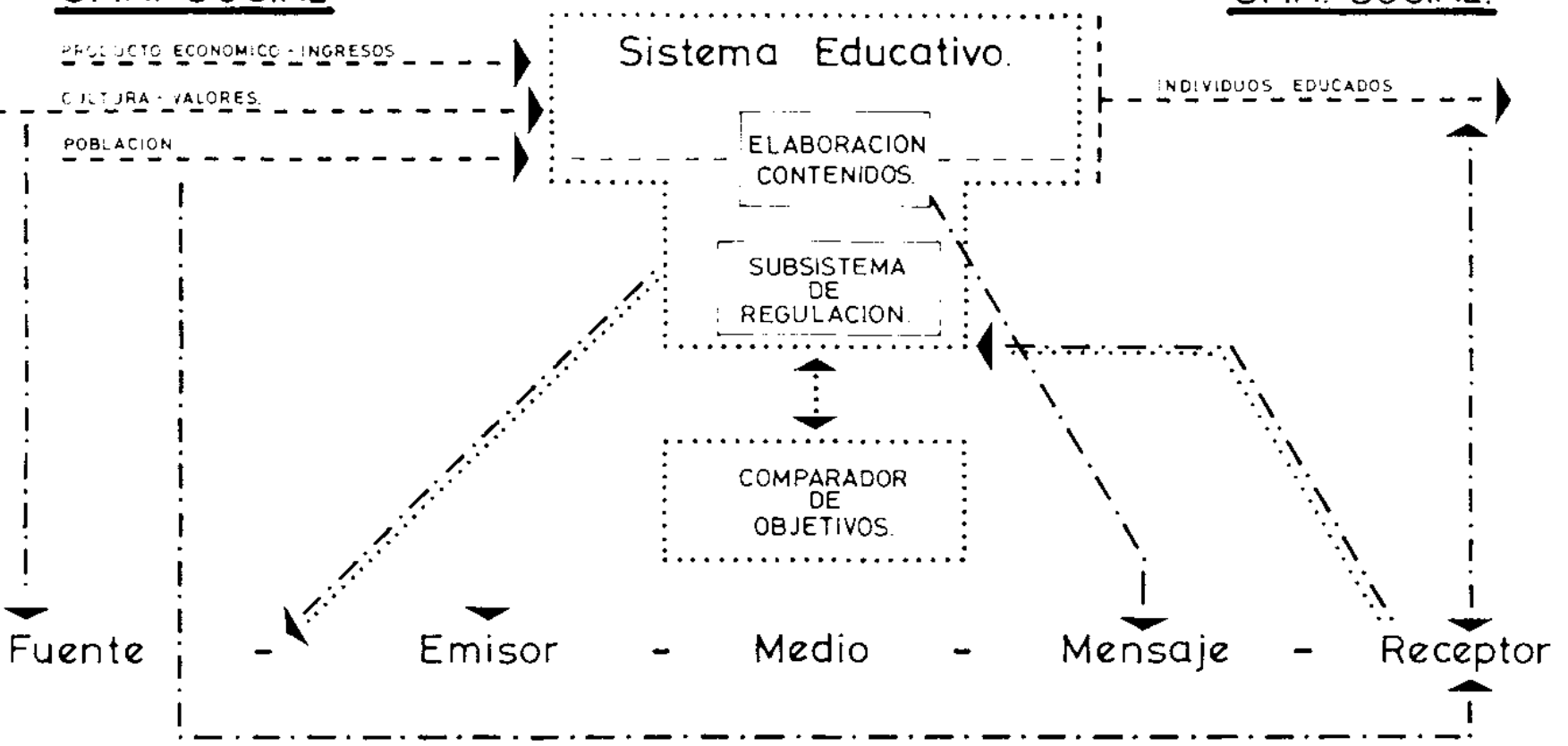

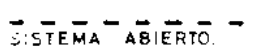

Sistema cerrado."

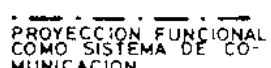


Considero que un sistema abierto en cuanto a intercambios de información (Valores, (ultura, Simbolos, Normas...) y de energía (recursos economicos, materiales... elc.) con su medio, y cerrado en cuanto a su autoregulación, tal como es al Sistema Viducativo, puede lalificarse de Adaptativo por ser abierto y de Cibernélico por ser cerrado su sistema regulador.

fil Sistema fiducativo es entonees un Sistema de tipo Adaptativo-(ibernetico to que implica poseer las características propias de este tipo do Sistema y por lo tanto unos mecanismos de funcionamiento plenamente parcjos.

Ii Sistema liducativo como hemos visto posec capacidad retroactiva o reguladora en aras a alcanzar las metas que le impone el Sistema Social. Es adaptativo por sulrir constantemente inferencias del medio o intentar mediante sus procesos reguladores responder a ellas ajustando su comportamiento a las exigencias del medio. lis además un Sistema de Información de Comunicación lat como se ha analizado, poscyendo por último eomo todo Sistema de este tipo, capacidad neguentrópica por recibir inlormación y "variedad" del medio social que lo envuelve.

Considero que donde reside lo original del estudio del Sistema Fiducativo no es tanto ser "sistema en si", cuanto ser sistema que se relaciona con la sociedad. fil verdadero papel, estado y funciones que desempeña el Sistema Educativo se descubrín en el estudio de las relaciones que mantienc con el Sistema Social. 1) ahí que si la Sociología de: la liducación pretende estudiar el Sistema liducativo, la esencia de su objeto será un estudio relacional. Por tanto considero que el verdadero e innovador estudio de los Sistemas liducativos, se llevará a cabo cuando próxinamente acometa cl estudio de las relaciones que como sistema mantione con el Sistema Social. Ia novedad del tema me obliga a considerar el presente estudio como paso previo para lograr próximamente el objetivo propuesto.

\section{3. Caracteristicas del Sistema Educativo}

\section{3. 1. Componentes del Sistema Liducativo}

los componentes del Sistema liducativo lo formaran el conjunto homogéneo de acontecimientos de carácter educativo así como los elementos que lo posibilitan en una sociedad determinada.

Algunos autores fijándose sobre todo en los elementos formadores del Sistema Fducativo intentan establecerlos y determinarlos. Por lo general la crítica que: considero puede realizarse a estos intentos es que bajo el sobrenombre de "Sistema Educativo" incluyen aspectos típicos de la liducación formal (o escolar). Veamos comparativamente para refrendar esta opinion las clasificaciones que presentan Ph. Coombs y. F. Mascort ${ }^{30}$

30 Viase respectivamente: COCMHBs, PH.: La Cirisis Mundial de: la Educación. Opus. Cit. Pes. 18, Crifieo I.

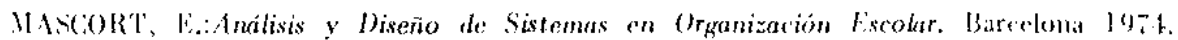

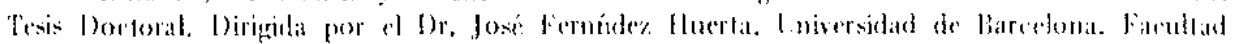

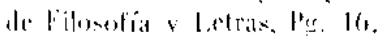


I. . Propositos y Prioridades

2.-.. Fistudiantes

3. Hirecoion

4. Listructura y Horario

5. Contenide

6. Protesores

7. Material I)idáctico

8. liscuelas y Ldificios

9. Ternologriat

10. Controles cualitativos

11.--Investigación

12. Costos
1. Ohijetivos

2. - Dirección (Management)

3.. Profesorado

4.- Contenidos

5.-- Estructura y Plan

6. Instrumentos de Medida

7.- Ayudas didácticas

8. Apoyos Materiales

Te las sistemáticas precedentes extratemos las conclusiones siguientes:

-Ia sintesis de li. Mascort no supone ninguna aportacion original al tema, puesto que sus elementos se hallan integrados a los formulados por Ph. Coombs.

- Ambos antores determinan los elementos componentes atendiendo más a un Sistema Escolar que a un Sislema liducativo.

A. Sanvisens basándose de alguna manera en Ph. Coombs ${ }^{31}$ ofrece una sintesis mas generalizada annque algumes de los clementos que cita como componentes del Sistema bducativo sean típicamente escalares.

Considera que el Sistema liducativo como proceso socio-cultural ofrece:

Finalidades

Hducandos en sus varios niveles

- Educadores, Maestros, Profesores

Grgano de Mirencion

Contenidos de la liducación

Material Inidáctieo

-. Tecmologria Fducativa

Centros fiducativos

l'anes y horarios

Controles roaliativos y emantiativos

Asperto investigador

Appecto eronomicos y administrativo

Por mi parte hasare la presentation de componentes del Sistema diducalivo atendiende a que todo Sistema, y por supuesto el que nos ocepa, está compueste por Infor-

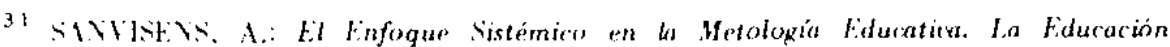
como Sistema. Opuso. Cit. Pg. 273. 
mación; Información que se desarrolla a partir de unos acontecimientos y a travén de. mos elementos.

Considerando entonces a la lnformación como elemento componente del Sistema liducativo, y a los acontecimientos y a los elementos como manifestaciones de la misma, considero que un Sistema lidurativo tal como ha sido definido y delimitado comprende:

\section{A. ACONTL: IMII:NTOS}

A. I. Información para el establecimiento det paradigrma o modelo normativo y axiológiro a aplicar a los individuos.

A. I. I. Objetivos de la Fiducación.

A. 1.2. Politicas liducativas.

A. I. 3. Alternativas liducativas.

A.2. Información para el establecimiento de la operatividad del sistema liducativo.

A. 2. 1. (Objetivos concretos a operativos de la educación (Contenidos).

A. 2. 2. Planes de Fiducarión.

A. 2.3. Programas de liducacion.

1. 2. 4. Presupuestos de la liducación.

^. 3. Información para la $\Lambda$ eción Fiducativa.

A. 3. 1. Asignación de recursos.

A. 3. 2. Coordinación de recursos.

A. 3. 3. Supervisión de los Recursos.

A. 4. Información para la lijecución Educativa.

A. 4. 1. Procedimintos Educativos.

A. 1.2. Instrucciones liducativas.

13. ELEMENTOS

B. 1. Vilementos Agentes de la liducación.

13. 2. Flementos lacientes de la liducacion.

B. 3. Fementos Materiales en los que se asienta la Kducación.

B. 4. Vehieulos materiales en los que se basa la bidaracion.

B. 5. Vehiculos simbólios en los que se hacia la liducación.

Por último en la base de toda romposición sistémica (y por tanto tambien en el Sistema bducativo), como romponente artutinador de los anteriormente mencionados y como fenómeno posililitador de la propia arción sistémira. nos hallamos con los fencmenos procestales-interactivos. Ln muestro caso dará lugrar a:

13. 6. Interaceión liducativa

Con ello ronsidero se sistematioa una lasilieacion de los componentes del Sistema fiducativo que puede aplieane tru general a cualquier fenóméno educarional ademas del merantente escolar. Por otra patrte esta clatnificarion me asienta sobre lom 
componentes esenciales de loda complejidad sistémica: Ia Información y los elementos que condicionan o participan de dicha información.

\section{3. 2. Estructura del Sistema Educativo}

En función de lo deterninado como componentes del Sistema liducativo la structura de dicho sistema comprendera:

-Los Actores de la Fducación (Fducadores-Fducandos) que se entregan a tareas y actividades diversas de indole educativa a partir de la:

-Interacción o comunicación relacional-educativa entre ellos. Fsto supone:

-... Ia Listencia de diversos status-roles en función de los papeles y posiciones que los actores asuman.

-..lista división de papeles y posiciones obliga al establecimiento de unas redes de relaciones socio-educativals.

-A partir de estas redes de relaciones socionducativas se formalizan los marcos o grupos educativos, tales entre otros como, los:

$\begin{array}{ll}\text { P'amiliares } & \text { Sexuales } \\ \text { Parentales } & \text { Estatales } \\ \text { liseolares } & \text { De lenguaje } \\ \text { De Amiges } & \text { Religiosos } \\ \text { De lidad } & \text { Polílicos } \\ \text { Iaborales } & \text { lite. }\end{array}$

Las relaciones a las que nos hemos referido posibilitan dentro de estos grupos la aparición de jerarquías.

- Por otra parte las actividades educativas manadas de la interación necesitan:

Condiciones lísicas

Condiciomes Materiales

- Condiciones financieras

- Condiciones Iemporales

- Medio Adecuado

Basamento de indole. Pedaróngica

\section{3. 3. Función del Sistema Fducrativo}

Muchas son las funciones que sergún los diversos autores realiza la liducación. Personalmente considero como fundamentale's las signientes: 
Fumion Conservadora o Reproductiva. ${ }^{32}$

fustion Creadora o Renovadora (Innovation). ${ }^{32}$

liunción Socializartora.

lianción Regularizadora del Hodo de Comportamiento.

linsenanza do: Pautas de Conducta.

Rápida y Eficaz Educación Personal.

listabilización Social.

Homogeneización Social.

Diferenciación y Selección Social.

Integración Social.

Transmisión Cultural.

Desarrollo de la Personalidad.

Imposición del Poder.

Promoción Social.

Promoción de la Investigación.

l'uede: decirse que la funcionalidad educativa es eminentemente social o al menos, con finalidad social aunque incida directamente sobre la estructura psiquica del Sujeto. El estudio de la funcionalidad del Sistema Viducativo se desarrolla a partir de las relaciones que como tal sistema mantiene ton la sociedad que de alguna forma al englobarlo lo condiciona. Nhora bien, si st tiene presente la característica de Sistema Abierto que a su ve\% posee el Sistema liducativo, éste también puede influir en la Sociedad. De hecho a través de las actividades anteriormente citadas el Sistema liducativo pretende cumplimentar su más importante actividad funcional: He refiero a la constante adaptación que ha de realizar con el ristema Social para asi anular los posibles desajustes funcionales. Sin embargo y como es natural, no siempre se comsigrue el perfecto acoplamiento; muy al contrario, en algunas ocasiones los sistemas liducativos se hallan desfasados de la realidad social que los envuelve entablándose entonces un conflicto dialéctico que puede alcanzar proporciones importantes, l'iénsese sino con la contestación de la juventud universitaria, basada en muchas ocasiones en al anquilosamiento que dichas instituciones sufren en comparación con el devenir del progreso social. til Sistema liducativo posee pues unas funciones básicamente sociales centradas en la adaptación que como subsistema se ve: obligado a ejercer con el Sistema del cual depende. Eillo no quita que se establezcan, en el intento adaptativo, situaciones no sólo de desajuste sino incluso conflictivas que sin embargo, ayudan a reestructurar a

32 Segin el sentido que esta terminología posee para BOULDIEL, P. \& PASERON, J. C.: la Reproductión. Elements pour une Theorie du Systeme d'enseignement. Paris 1970, Edit. Minuit.

Sobre esta obra véase:

DIOX, H.: Sociolegía a Adeología. Bareclona 197.t. Fidit. Fontanella. Libros de Confrontación. Serie . vociologica, $11 .{ }^{\circ}$ 4. pgs. 123 a 139. 
ajguno de los sisternats (at rocial o el Piducativo) posibilitando en consecuencia, o bien la eficarial fumeional, o bien el strgimiento de nuevats funciones a realizar que puedern ser de nuevo fuente y origen de conflicto.

J. Paredes considera esencial lo que denomina "Funcion de Producción del Sistena kducativo":

"lit sistema liducativo está llamado a producir la tranformación continua de las relaciones de producción en los sistemas encargados de las mismas..."

"li tistema liducativo ha de atender no sólo a la transtormación de las relaciones de producción de los demás sistemas produetivos sino sobre todo a su propia transformación de las relarione's de producción. ${ }^{33}$

Tambien se ha querido ver al listema liducativo con lit funcion primordial de controlar los diversos aspectos sociales:

"Fl proceso educativo es a conducto por ef que la Sociedad transmite su cultura a las generaciones siguientes. Fil objetivo social de la liducación es inducir a las personas a aceptar las más altas normas de combeta gue posec la cultura y a comformarse en ella. Actía por medio de Sintemas formales y de procedimientos intormales pero su producto definitivo es una persona que conoce la diferencia entre las conductas aprobadas $y$ desaprobadas $y$ que puede ocupar su puesto como miembro que "este conforme" de la
fociedad" 34

Listos procedimientos de control se ejercen a través de: los siguientes instrumentos educativos:

-Selección de los Mensajes liducativos.

- Análisis de la realidad a la que se apticarán.

- Predicción de la situación que se pretende.

- Fstablecimiento de estrategias de Control:

C... Sobre los medios de Fducacion.

- Sobre los agentes de liducación.

-Sobre los sujetos objetos de la tiducación.

Control sobre recursos lísicos, conómicos y materiales.

P'or lo general sucle ser el Subsistema bducativo liscolar, y el Subsistema Educativo de los Mass Media, los que encabezan las funciones controladoras que pueden ser ejervids por cualquiera de las antoridades instilucionales sobre las que

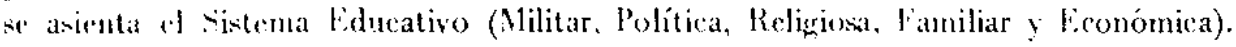

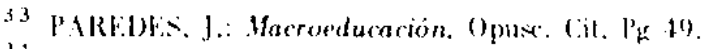

34 FlchlltR: Sociologia. Harcelona 1972. Edit. Herder. Pg. 382. 
Los fines que la sociedad persigue ejerciendo funciones controladoras a partir des Sistema liducativo son las sigrientes:

Seguridad.

jiquilibrio.

Orden.

Manteninniento de las Situaciones.

Adaptación ded Individuo al Nedio Fisice y Social. ${ }^{5}$

Sin embirego y en mi opinión, el querer ver la función de control en el Sistema lidacativo, es desfigurar su misión fundanental si al mismo tiempo no se menciona la actividad contraria a la de control que posece como propia y especifi(a. He refiero al hecho de que la liducarión no salvaruarda sólo Valores -y controla su inclusión en el contexto social sino que también y al mismo tiempo presupone nuevos contenides axiologiress que expande hacia aluera, hacia la socicdad. Contemplar la funcion de control en el Sistema liducativo es simplemente observar la mitad de un procese circular que: puede culminar con la inclusión en la siociedad de nuevos valores y paradignsas engendradores de acción social y por

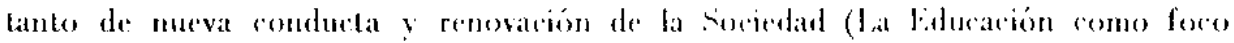
neguentrípic‘o).

lil problemat del Control o de la musevación es frute de las situaciones

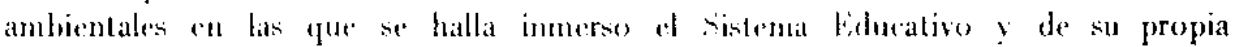
siluación interna que posibilitará una acción arlaptativa de tipo retroactivo (Control) o de tipe propulsor de la acrion (leed-before u optinización de la inno. valion).

\section{3. 1. El "Proceso" del Sistema Educativo}

Como ya dijimos a hecho educativo surge ton la Sociedad. Su evolución es paralela al desarrollo que en complejietad va adquiriendo la propia sociedad, hasta que llega un momento en el que la sociedad va treamdo y amplando los mecanismos basiose de indole educativo. Hoy en dia ex sistema liducativo es eminentemente amplio y complejo incidiendo en la mayoria de grupes serciales que se conforman en la sociedad. Como die: Nieves Aponte." "." proceso educativo es uno de los procesos cardinales primigenios y vilales de tuda cultura: es tan fundamental que presiona en toda sociedad..." lo cierto es que esta presion no sólo es horizontal y sincrónica, sino que también ha sido y sera una ronstante vertical diacronica especilicamente historiea y evoluliva. "la liducacion es siempre una funcion de una civilización en particular en ma epoca texpecifica de la historia".37

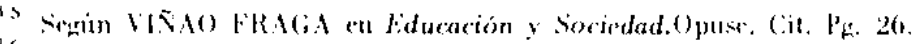

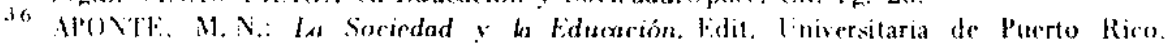

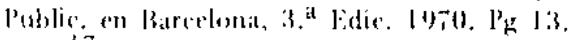

17 tbitem. Pg, \$6. 
Atendiendo a esta realidad procesual de indole diacronica he intentado sistematizar en esquema unas bases para lo que en un pestrer desarrollo -.. podría ser un estudio sistémico-evolutivo del proceso que la liducación ha mantenido en su dialéctica con la Sociedad.

Considero cinco fases prineipales, la última de las cuales corresponderá a la actual, que analizaré a partir de otras tantas variables:

-Objetivo del Sistema Fiducativo.

-Aspecto a desarrollar por el ristema fiducativo.

- Estructura organizativa del Sistema.

- Tlipo de dirección sistémica.

- Valores que promulga el Sistema.

PROCESO DEL SISTEMA RIDUCATIVO (VISION DIACRONICA)

\begin{tabular}{|c|c|c|c|c|c|}
\hline $\begin{array}{l}\text { VARIABLE: } \\
\text { CONSIDI:RADA } \\
\end{array}$ & $\begin{array}{l}1^{\alpha} \\
\text { PASt: }\end{array}$ & $\begin{array}{l}22^{\alpha} \\
\text { FASE }\end{array}$ & $\begin{array}{l}37^{2} \\
\text { JASE: }\end{array}$ & $\begin{array}{c}4 i^{2} \\
\text { FASF: }\end{array}$ & $\begin{array}{c}S_{1}{ }^{3} \\
\text { J.AS1: }\end{array}$ \\
\hline $\begin{array}{l}\text { (H)yetives tel Sinte } \\
\text { ma Educstivo }\end{array}$ & 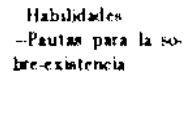 & Urtodexia tio ia fe & $\begin{array}{l}\text { Idaplación politico } \\
\text { vocial }\end{array}$ & $\begin{array}{l}\text { Consolidación de } \\
\text { las histructuras sis. } \\
\text { ciales } \\
\text { (entratifirarión } \\
\text { actual) }\end{array}$ & $\begin{array}{l}\text { Avatce y dexarrullo } \\
\text { Social }\end{array}$ \\
\hline $\begin{array}{l}\text { Ampercto a deserro } \\
\text { lar }\end{array}$ & Producrion & $\begin{array}{l}\text { Flfiriencia de ba } \\
\text { Fdurarion }\end{array}$ & $\begin{array}{l}\text { Expansión de to } \\
\text { Fiducacxion }\end{array}$ & 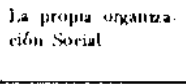 & $\begin{array}{l}\text { Kesolución de } \\
\text { P'roblembar } \\
\text { Gunovackísn }\end{array}$ \\
\hline $\begin{array}{l}\text { Eonnciura Urzani- } \\
\text { cative del Sintemin }\end{array}$ & Famtist & $\begin{array}{l}\text { limintraliesia Verti- } \\
\text { ral }\end{array}$ & Cemtralizatla & $\begin{array}{l}\text { Directiva } \\
\text { Cinipess de Ilirre. } \\
\text { cion }\end{array}$ & 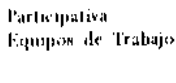 \\
\hline $\begin{array}{l}\text { Tipe de Direcrion } \\
\text { Siuternk'a }\end{array}$ & Paternal & Dherctira & 1)ekgation & Vuquilante & Parlicipativa \\
\hline $\begin{array}{l}\text { Valixes que pro. } \\
\text { mulore el Sistemis }\end{array}$ & Miticox & Jielisiteximen & Peolílseses & Ficonersicon & Tecnolúgicos \\
\hline
\end{tabular}




\section{CONCILSION:}

\section{MODHLLO DLI SISTHMA FDUCATIVO}

l'n modelo de un sistema liducativo concrelado en una suciedad determinada deberia istudiar:

Flujos de liducandos.

- Flujos de Fidueadore's.

- Vecesidades y optimizacion de codificios y bienes mucbles a inmuebles an general.

- Flujos de coste y linanciación.

Concreción del numero de individues educados y especializados de actuerdo con el desirrollo social y los puestos de trabajo.

Determinación general de las neresidades sociales de la liducación.

Política de liducacion optima.

Asignación de recursos.

Programas de Enstinamzatiducación optima.

Modelos de asperesos del Sistema liducativo ya han sido llevades a la practica con una formalization cuantitativa nada desdenable.

Sin embargo estos nodelos solo se refieren al subsistema fiscolar, dejando et resto; del Sistema liducativo sin tratar. Lor más importantes son posiblemente los siguientes:

lil Modelo de Itertor (orrea: Modeles Mathematiques pour la planification de lenseznement, puldieado en Paris por la (). (:. li. l). li.

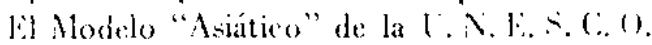

lin lispana: El Hodelo Español de Desarrollo Educatimo,publicado an Madrid por el Ministerio de liducación y Ciencia.

fin todo sistema fiducativo se da de alguna manera los tres asperetos siguientas:

1. Control solure la accion del sistema.

2. P'rogramas-l'royecto de kesializatión (o fenomeno de indole educativo-social lípico del Sistema liducativo).

3. Práctica de dicha fenomenología educativo soriat.

lin los grátices que inchimos a contintasión representamos la "dirección" de. estos tres procesos asi como los elementos gue los posibilitan teniende en cuenta que las relations que inclusen por ser sistemicas son tal como hemos visto de indole informational. 


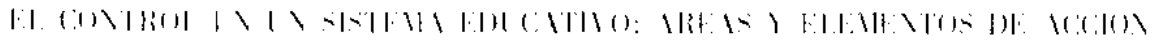

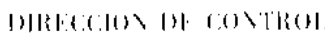

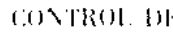

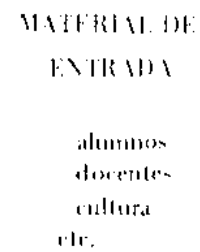

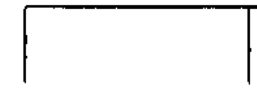

(1) $11+13)(1)+2$ ind indis

\section{Birend Btidlat} $\therefore$ ististritlal limelal

limilia ite.

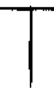

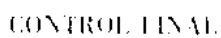

Cialidial ate:

lat Sortalizactum

feducartion recibsta

fopritus somantario 116.

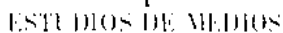

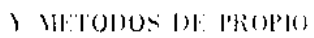

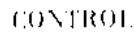

Campramals lilerteriales

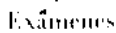

lasestigasion ato atutiolomes "lis.

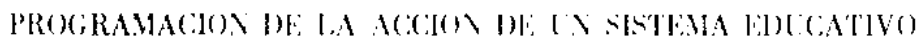

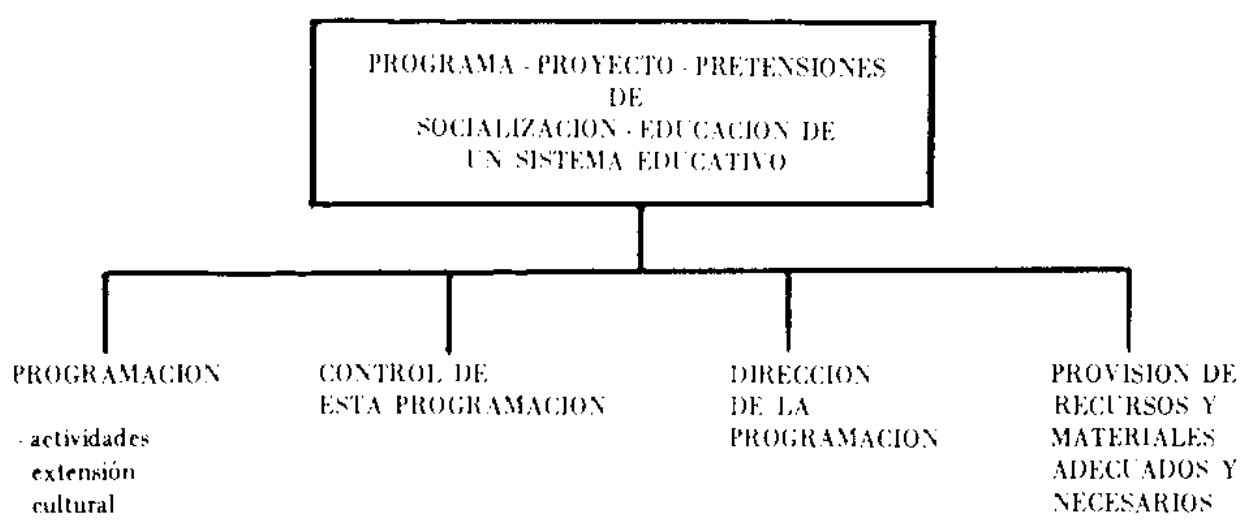

-Conferencias,..efts.

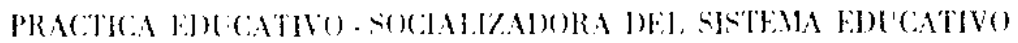

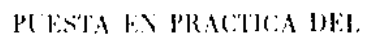

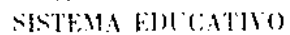

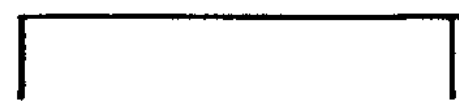

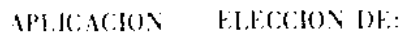

(1) los

- Tiempos

phocikg.11:5
- Nementos

siajclor

() pertunidades

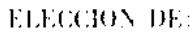

Protagonislas:

Persomas

linnicas

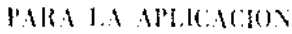

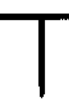

listolos ats.

VIFIS postosi.11) (11)

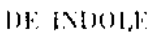

bolcotis
(CONOCIMHENTO IBE Kliste'Tdoos polk

I.AS:

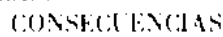

(o) 
El diseno anterior, expresado a nivel tripartito, pucde aplicarie a las situaciotes expresadas y que concurren en un Sintema siocial que pretendiese a través de su Sistema liducativo realizar un proyecto de cambio o innovacion socio-rultural.

Resumiendo lo anteriormente expresido y de forma procesual diseñariamos el Sintemat liducativo de la siguiente forma:

BNTKADAS

$\therefore 1111) .12$

Situacion de

(ntories

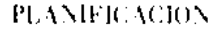

Situaciones que

afectaran la

siluación interna
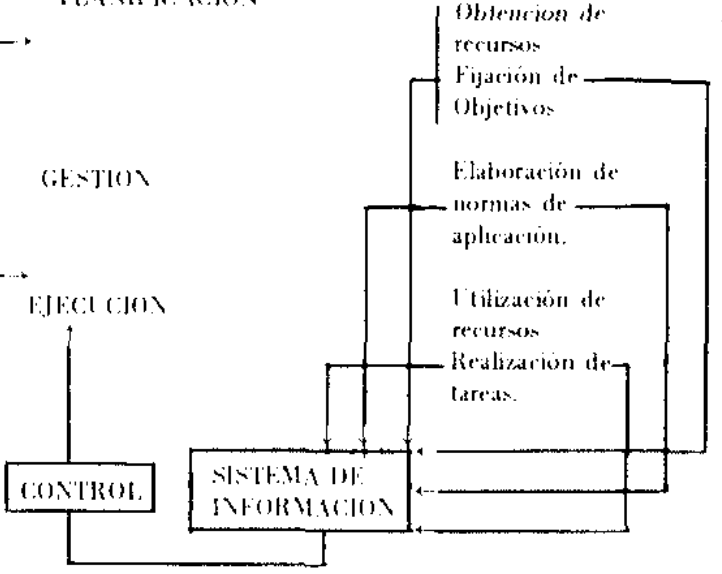

Hist

politicon

enoliter

ere.

firmese

Jorsisomes

P'rucedimantos

Irtuation

E.jecucion

Riralizackis

Con ello considero posecmos una bise para pestular los principios en que se asienta ef sistema lidueativo ${ }^{38}$ cuya formulation se basaria en los siruientes paradigmati:

1. los Procesos de P'roducción-sintesis y comsiguiente resultado de los medios y métodos de produccion conforman ha base que estructura la acción humana.

2. Jistas acciones humanas crean los valores, las nomas y el mundo de la cultura y de los paradignas de significaciones y simboles.

3. Listos valores, normas y significacomes simbelieas asi eome la cultura en general puede ser $y$ de hecho es activada, estructuradat y ordenada en compartimentos cientifico-culturales.

1. La transmisión de estos hagrajes culturales bien sea a nivel intelectual, bien a nive! de pautas de conducta, posibilitan la socializacion (integration en la sociedad) de los individuos que la forman.

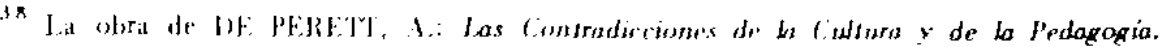

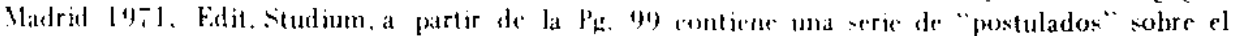
sistema Faduation que no quisiera se confundiesen ene he que formulo, fruto original de la sintesis yue presento. 
5. La Organización Social se preocupa de dicha transformatcion; es lo gue se denomina Sistema Educativo.

6. El Sistema Educativo se hasa entonces en la interacción de información "socializante-educadora".

7. El resultado de esta interacrión cuyo fruto es la socialización supone contemplar una sociedad compartimentada en niveles diferenciados de indole intelectual, conomico, de prestigio y de consenso social.

8. Fista concepción selectiva supone la posibilidad de integración de cada individuo en el seno de la sociedad a partir de su adscripción a un status-rol determinado $y / 0$ al mismo tiempo ser fruto de tensiones y por consiguiente de conflicto social.

lil mecanismo de la dinámica del Sistema liducativo y me refiero ahora a su comportamiento teniendo en cuenta su dependencia con el Sistema Social- lo vemos centrado en los siguientes puntos:

1. Li Sistema bducalivo se halla "adecuado" dentro de la dinamica de "cambio de estado" del Sistema Social.

2. El Sistema liducativo mantiene su constitución dentro de unos determinados limites que vienen mareados por la ardaplatión gue posee en el sistema social.

3. I a Organzacion del Sistema liducativo desarrolla gracias a su capacidad retroactiva un tipo especial de sensibilidad selectiva hacia los fenomenos internos que en el acontecen o la influencia que ejerce sobre el Sistema Social.

4. Fin consectencia los focos sensibles del Sistema bducativo son capaces de: reconocer hasta que punto los cambios que sufre se hallan en relación (o desviados) con los objetivos que posier.

5. Cuando en un Sistema liducativo la retroaccion no logra armonizar la conducta deseada se produce o bien una correceión por parte de] Sistema Social o bien un foco de desviación gue puede modificar ciertos aspectos esenciales o no del Sisterna Social.

6. Un Sistema lielueativo no sólo es un Sistenta Abierto al Sistema Social sino que funcionalmente actúa como un sistema de Comunicación que relaciona la cultura de la sociedad con los individuos de la misma.

7. Todo Sistema Edurativo por complejidad interna y por sus variaciones en el medio social fruto de su estructura sistémica de tipo abierto-cerrado puede aportar posibilidades de variación a su propia conducla (desviación).

8. In Sistema bdueativo pose en funcion de sus elementos que lo componen eriterios selectivos que permiten entrealcar de sus posibilidades de variación, ha más adecuada a las circunstancias que el Sistema rocial le exija (retroacción).

9. Fil Sistema Social reviste al Sistema faducativo de la capacidad dispositiva a mantener la conducla o d comportamiento elegido si este e's exiloso.

10. El Sistema liducativo por ser ahierto e intereambiar información con el Sistema Social es de por si un Sintema Veguentrópies. Además por su comportamiento como sistema de comunication y por la manipuación que de elementos informativos realiza, el Sistema Educativo se presenta como un foeo de neguentropia y por tanto como revitalizator del Sistema Social. 
11. Fin consecuantia un ristema fiducativo nunca por ser neguentropico puede ser considerado como un sistema an rquilibrio. Además por ser foco de neguentopia del Sistema Social, ofrece a ente posibilidades de variarión y rambio.

12. lil Sistema Fdurativo por ser un Sistema neguentrópico del bistema Social es un sistema de redes de commenication de información que mantiene sat pervivencia relacional con el Sistema Social.

13. In Sistema liducativo manticne: una cohesión e interdependencia que se: halla en función de la cantidad y variedad de información que recite y manipula.

14. I a estructura del Sistema fiducativo es un complejo de redes de comunicación de infonnacion entablecida entre los diversos elementos que componen al sistema.

15. I at relación existente entre los diversos núcleos o subsistemas de un Sistema liducativo y entre aquellos y este se basan en of intercambio que de informacion mantienen y en la información que del Sistema social reciben.

16. La operatividad del sistema liducativo es fruto de los procesos de información que contiene y que mantiene con el sistema tocial.

17. Ios fenomenos correctores o retroactivos que el sistema fiducativo recibe a nivel superestructural (del tistema Social) se hallan en función de las salidas que el Sistema liducativo le ha proporcionado.

18. Todo Sistema liducativo posete unas reglas que regulan la informacion que: se transmite dentro de il. listas reglas vienen dadas por las caracteristicas psiquicas-personales y materiales de sus elomentos lo que hace se distingan diversos roles, niveles... etc.

19. Ia elección de la conducta adaptativa al sistema social que realiza el Sistema liducativo se halla en función de los insumos " entradas que recibe de aquel y de la "adidad" de las salidas que le ofrece.

20. La unidad hásica de análisis de un sistema Educativo es la informacion fruto de la relación interactiva que se establece entre sus elementos.

lin defunitiva puede decirse que las caracteristicas analiticas que diseñan un Sistema fiducativo se hallan en función de las que posec come Sistema Abierto Ce. rrado y de comunicación, asi romo por la relación que como sub-sistema mantiene con rl Sistema Social. 
